\title{
Full retroauricular skin and fascia expansion in microtia reconstruction: a single center experience of 166 cases
}

\author{
Ji-Hua Wang, Ting Tang, Yong-Jing He, Ying-Jia Zhang, Yun Yang \\ Department of Plastic Surgery, the Second Affiliated Hospital of Kunming Medical University, Kunming 650101, Yunnan, China.
}

Correspondence to: Prof. Ji-Hua Wang, Department of Plastic Surgery, the Second Affiliated Hospital of Kunming Medical University, Dianmian Great Road, Kunming 650101, Yunnan, China. E-mail: wangjihua1966@163.com

How to cite this article: Wang JH, Tang T, He YJ, Zhang YJ, Yang Y. Full retroauricular skin and fascia expansion in microtia reconstruction: a single center experience of 166 cases. Plast Aesthet Res 2016;3:364-7.
Article history:
Received: 21-09-2016
Accepted: 04-11-2016
Published: $30-11-2016$

\section{Key words:}
Microtia,
expansion,
autogenous costal cartilage,
ear reconstruction

\section{INTRODUCTION}

Ear reconstruction with autogenous rib cartilage for congenital microtia is a challenge for plastic and reconstructive surgeons. In addition to the surgeon's

\begin{abstract}
Aim: Ear reconstruction is a challenge for plastic and reconstructive surgeons. The ear requires sufficient skin coverage and a three-dimensional (3D) cartilage framework. In this paper, the authors present their 10-year experience in microtia reconstruction using tissue expansion and an autogenous rib cartilage framework. Methods: Ear reconstruction was performed in 3 operative stages. During the first procedure, a 50-80 mL kidney or cylinder-shaped expander was implanted deep to the subcutaneous fascia of the retroauricular mastoid region. Over a period of 3-5 months, the expander was filled to a final volume of $80-110 \mathrm{~mL}$. In the next operation, the retroauricular fascia was eliminated or reserved following expander removal, and the autogenous costal cartilage framework was placed below the expanded skin flap. At the third and final stage, the earlobe transposition, tragus construction and conchal deepening were performed. Results: A total of 165 patients (166 ears) were reconstructed using tissue expansion and an autogenous rib cartilage framework. Complications included hematomas in 3 cases, expander exposure in 8 cases, cartilage exposure in 6 cases, infection and cartilage resorption in 2 cases, exposure of steel wire in 4 cases, and aseptic seroma in 2 cases. Follow-up ranging from 3 months to 5 years showed that 159 patients were satisfied with the reconstructed ear including size, location, projection, convolution, skin-colour matching, symmetry with opposite ear. Conclusion: Expansion of the retroauricular skin and fascia can provide sufficient non-hair-bearing skin and tissue for coverage of a three-dimensional costal cartilage framework. Avoidance and prompt treatment of complications are advised in order to obtain a satisfactory reconstruction of the ear.
\end{abstract}

skills, successful ear reconstruction is dependent upon a framework for the ear material and the overlying skin coverage. Autogenous costal cartilage has been widely used as a source of the ear framework. ${ }^{[1-4]}$ However, great success was not achieved until 2006

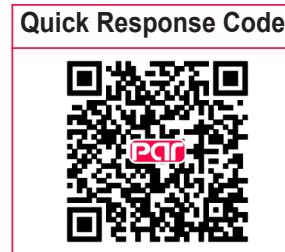


when a large series of ear reconstructions with tissue expansion were reported. ${ }^{[5,6]}$ At present, retroauricular skin tissue expansion is one of the most popular techniques for ear reconstruction. In this study, tissue expansion and autogenous costal cartilage were used for reconstruction of 165 patients with 166 cases of microtia. The majority of the reconstructed ears obtained a good contour with a low incidence of complications. In this paper, the authors report their experience with microtia reconstruction by use of fullthickness skin and fascia expansion and a threedimensional costal cartilage framework.

\section{METHODS}

From August 2005 to August 2015, 165 patients with 166 cases of congenital microtia were reconstructed using fully expanded retroauricular skin and fascia flaps combined with an autogenous costal cartilage framework at the Department of Plastic Surgery of the Second Affiliated Hospital of Kunming Medical University. Among these patients, 112 (67.9\%) were males and $53(32.1 \%)$ were females. Patient age ranged from 7 to 52 years (average 15.8 years). Of all patients, 110 cases $(66.7 \%)$ of microtia involved the right ear, 54 cases (32.7\%) involved the left ear, and 1 case was bilateral [Table 1].
Table 1: Basic information of 165 patients (166 ears)

\begin{tabular}{lr}
\hline Characteristics & $\boldsymbol{n}(\%)$ \\
\hline Gender & \\
Male & $112(67.9)$ \\
Female & $53(32.1)$ \\
Side & $110(66.7)$ \\
$\quad$ Right & $54(33.3)$ \\
Left & $2(0.60)$ \\
$\quad$ Bilateral & \\
Associated deformities & $5(3.6)$ \\
$\quad$ Hemifacial microsomia & $1(0.6)$ \\
Facial nerve weakness & $2(1.2)$ \\
Opposite ear deformity & $4(2.4)$ \\
Fistula & $3(1.8)$ \\
Accessory ear & \\
\hline
\end{tabular}

For the patient with left-side microtia [Figure 1A], during the first procedure, a 4-cm length incision parallel to the hairline was made on the scalp and a subfascial fascia pocket was dissected. After meticulous haemostasis was obtained, a $50-80 \mathrm{~mL}$ (corresponding to the size of the opposite auricle) kidney-shaped or cylinder-shaped tissue expander was implanted under the fascia pocket, with the valve of the expander placed in the subcutaneous tissue of the scalp or left externally. A negative-pressure drain was placed inside the pocket prior to closure of the incision. The suction drain and the suture were removed 6 days and 10 days postoperatively, respectively. Tissue expansion was performed twice
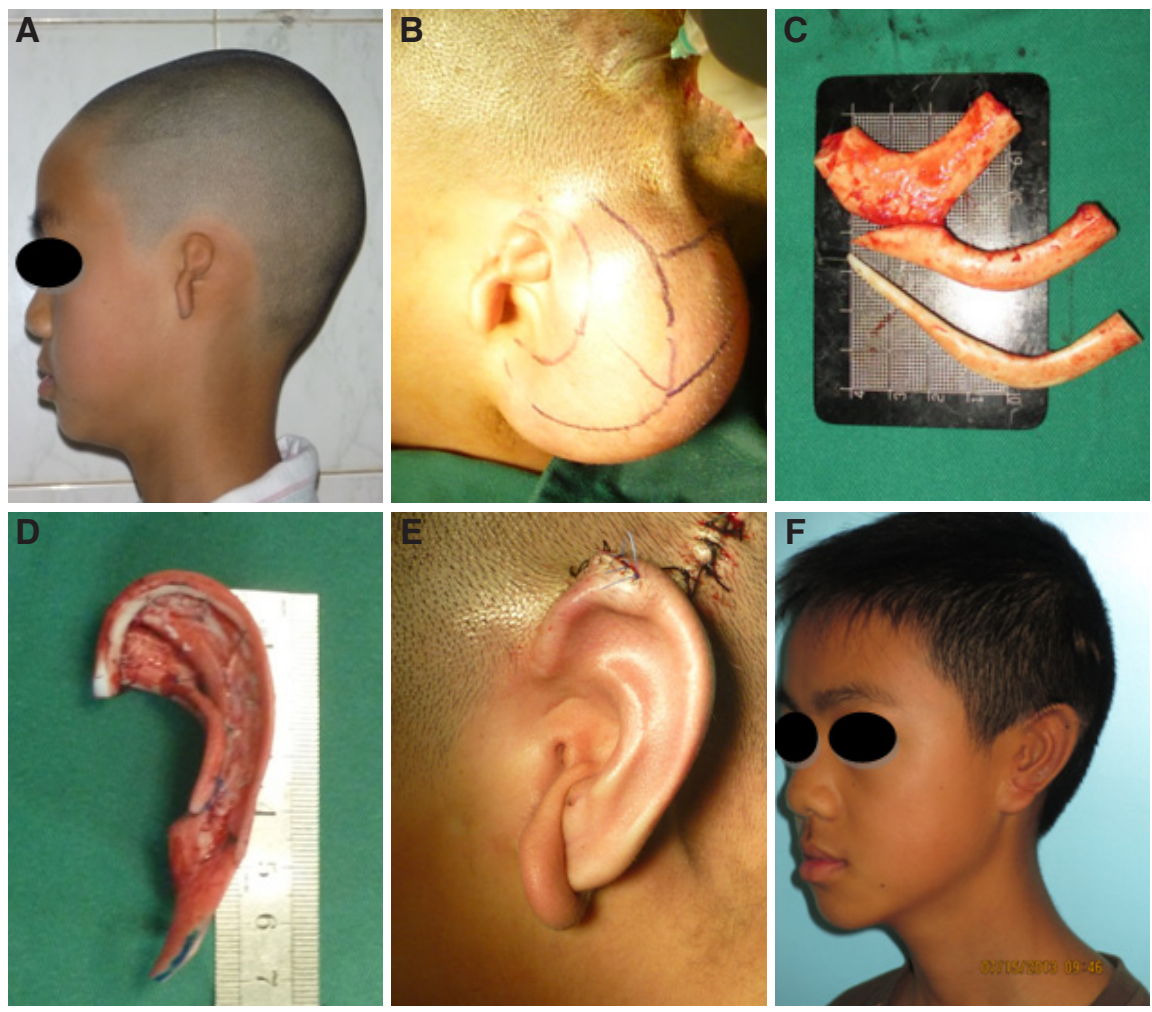

Figure 1: (A) The appearance of the left microtia; $(B)$ the appearance of the fully expanded tissue expander in the left retroauricular region; (C) the harvested costal cartilages; (D) three-dimensional (3D) costal cartilage framework; $(E)$ the appearance of the reconstructed ear immediately following application of the negative-pressure drain; $(F)$ the malpositioned earlobe was transferred and connected to reconstructed ear 
a week beginning 7 days after surgery and continuing until the targeted volumes were achieved [Figure 1B]. The second stage was performed following the completion of expansion. Preoperative computer tomography (CT) of the rib cartilage to be used for reconstruction was performed to assess for the degree of calcification and to measure rib cartilage parameters (length, width, and thickness). At the final surgery, the fascia was separated from the skin flap following removal of the expander, and the remnant ear cartilage was carefully dissected and preserved. Based upon the preoperative CT of the costal cartilage, normal costal cartilage from the seventh to ninth contralateral ribs were harvested by another surgical team [Figure 1C]. The harvested costal cartilages were then carved to include the structure of the ear, including the scapha, helix and triangular fossa [Figure 1D]. The redundant cartilage pieces were assembled to form a crescent-shaped pad and were inserted beneath the carved costal cartilage in order to enhance projection. The cartilage complex was assembled with 5-0 stainless steel wire and placed into the expanded pocket. The size, location and angle of the cartilage framework were adjusted until the reconstructed ear was consistent with the opposite ear. The drainage tube was inserted between the flaps and the cartilage framework. Finally, the shape of the reconstructed ear appeared after the drainage worked [Figure 1E]. The reconstructed ear was not covered dressing and a pressure dressing was applied to the retroauricular region. The dressing was removed on the first postoperative day to prevent infection. In order to maintain effective suction, the drain was evacuated every $2 \mathrm{~h}$ during the initial $24 \mathrm{~h}$ postoperative. The drain and sutures were removed at 6 and 10 days following surgery, respectively. Three to five months later, the malpositioned earlobe was transferred and connected to the reconstructed ear, the redundant cartilage and earlobe soft tissue were excised, and excess subcutaneous tissue was removed in order to deepen the conchal bowl [Figure 1F].

\section{RESULTS}

A total of 166 ear reconstructions were performed in 165 patients with microtia. There were three cases of hematoma, but expansion was successfully accomplished following evacuation. Exposure of the tissue expander occurred in 8 cases, which were performed ear reconstruction ahead of time using expanded skin and temporoparietal fasical flap with skin grafts. After the second stage, exposure of the cxf occurred in 6 patients, and was repaired by use of a local skin flap or temporoparietal fascia flap and skin graft. Infection occurred in 2 patients and was treated with systemic antibiotics. For these two patients on
Table 2: Complications in 165 cases

\begin{tabular}{ll}
\hline Complications & $\boldsymbol{n}(\%)$ \\
\hline Expander hematoma & $3(1.8)$ \\
Expander exposure & $8(4.8)$ \\
Exposure of cartilage & $6(4.3)$ \\
Infection and cartilage resorption & $2(1.5)$ \\
Fracture of upper pole of ear framework & $4(2.4)$ \\
Extrusion of steel wire & $4(1.80)$ \\
Sterile seroma & $2(1.5)$ \\
Pneumothorax & $1(0.61)$ \\
Subluxation of cervical vertebra & $1(0.61)$ \\
Hypertrophic scar & $5(3)$ \\
\hline
\end{tabular}

the third surgery of earlobe transposition, skin graft was performed ahead of time, which resulted in poor reconstructed ear because of partial absorption of cartilage. Sterile seromas occurred in 2 cases 2 weeks postoperatively. No seroma accured again after negative press suction. Fracture of upper pole of the ear framework occurred in 4 cases, creating a less satisfactory auricular contour. Extrusion of the steel wire occurred in 4 cases. Other complications included 1 case of pneumothorax, 1 case of cervical vertebral subluxation, and 5 cases of hypertrophic scars at the chest harvest site. All complications were successfully treated [Table 2]. The postoperative satisfaction rate was $96.4 \%(159 / 165)$.

\section{DISCUSSION}

Since Hata et al. ${ }^{[7]}$ initially used a tissue expander for correction of congenital microtia. Since then, ear reconstruction using an expanded retroauricular skin flap and autogenous costal cartilage has been widely used. ${ }^{[8-10]}$ Skin expansion provides nonhairbearing, thin and well-vascularized skin. However, a $3 \mathrm{D}$ framework and skin grafts are often required as well. ${ }^{[8,9}$ Liu et al..$^{[5]}$ and Zhang et al. ${ }^{[6]}$ have reported the use of 2 expanders for ear reconstruction without skin grafting. However, this method increases the complexity of the operation and increases the risks of complications associated with expansion, including hematoma, exposure, and an obvious post-auricular scar. Chen et al[. ${ }^{[11]}$ reported implanting a $50-\mathrm{mL}$ kidney-shaped expander in the retroauricular mastoid region and infusing saline solution to a final volume of $100-120 \mathrm{~mL}$. In this fashion, sufficient retroauricular non-hair-bearing skin was obtained for coverage of the auricular cartilage framework without the use of skin grafts or a retroauricular fascial flap. This method of over-expansion nonetheless risks complications including exposure, skin necrosis, and possible elongation of expansion time, or even failure of expansion.

In order to reduce the complications mentioned above, we implanted a 50-80 mL expander beneath the retroauricular fascia to reduce the risk of exposure, and infused saline solution for a total volume of $80-110 \mathrm{~mL}$ 
to fully expand the fascia and skin. The region of nonhairbearing skin in the mastoid is relatively small, and therefore an $80 \mathrm{~mL}$ expander was selected. At the second stage operation, the integrated fascial flap was dissected and covered to the cartilage framework, only imperfect fascia was removed. The thickness of the expanded skin flap was approximately $1.5 \mathrm{~mm}$ through treated, it can wrap erect 3D framework and reproduce reconstructed auricle substructure.

Harvesting ipsilateral rib cartilage has been recommended by Nagata ${ }^{[4]}$ and Firmin. ${ }^{[12]}$ Dashan et al. ${ }^{[13]}$ routinely harvested rib cartilage from the right side in order to protect the left chest wall and the heart. In the current study, contralateral costal cartilage was routinely harvested to take advantage of the natural bend and tortuosity of the contralateral costal cartilage. After nearly 3 years of observation, there were no side effects to donor site. In this study, a two-dimensional X-ray template combined with a 3D plaster model was used to fabricate a cartilage framework similar to that of the opposite normal auricle. Jiang et al. ${ }^{[14]}$ have reported the use of four different methods to sculpt the cartilaginous framework. In our patients, we used 3-4 layers of costal cartilage to fabricate the framework, which is similar to Jiang's technique.$^{[14]}$ In adult patients, the seventh rib cartilage was divided into two parts, with the external portion used for the helix and superior crus, and the internal portion used for the main body of the framework, the other cartilage were used for base to increase protrusion.

Stabilization plays an important role in construction of the cartilage framework. Therefore, each part of the framework, and in particular the helix, must have sufficient thickness. The 5-0 stainless steel wire was used to suture and fix all parts of the cartilage framework.

\section{Financial support and sponsorship}

This work was financially supported by the grants of National Natural Science Foundation of China (No.81660319), and Health Science and Technology Plan Projects of Yunnan province (No.2016NS266).

\section{Conflicts of interest}

There are no conflicts of interest.

\section{Patient consent}

The patient gave full written consent to the use of images for publication.

\section{Ethics approval}

We declare that our research was carried out in a high ethical standard, and the research protocol was approved by our local Ethical Committee.

\section{REFERENCES}

1. Tanzer RC. Total reconstruction of the auricle: a 10-year report. Plast Reconstr Surg 1967;40:547-50.

2. Brent B. Ear reconstruction with an expansile framework of autogenous rib cartilage. Plast Reconstr Surg 1974;53:619-28.

3. Brent B. Technical advances in ear reconstruction with autogenous rib cartilage grafts: personal experience with 1200 cases. Plast Reconstr Surg 1999;104:319-34.

4. Nagata S. A new method of total reconstruction of the auricle for microtia. Plast Reconstr Surg 1993;92:187-201.

5. Liu J, Sun J, Li X. Total auricular reconstruction without skin grafting. J Plast Reconstr Aesthet Surg 2011;64:1312-7.

6. Zhang GL, Zhang JM, Liang WQ, Chen YH, Ji CY. Implant double tissue expanders superposingly in mastoid region for total ear reconstruction without skin grafts. Int J Pediatr Otorhinolaryngol 2012;76:1515-9.

7. Hata Y, Hosokawa K, Yano K, Matsuka K, Ito O. Correction of congenital microtia using the tissue expander. Plast Reconstr Surg 1989;84:741-51; discussion 752-3.

8. Jiang H, Pan B, Lin L, Cai Z, Zhuang H. Ten-year experience in microtia reconstruction using tissue expander and autogenous cartilage. Int J Pediatr Otorhinolaryngol 2008;72:1251-9.

9. Park C. Subfascial expansion and expanded two-flap method for microtia reconstruction. Plast Reconstr Surg 2000;106:1473-87.

10. Zhang Q, Quan Y, Su Y, Shi L, Xie Y, Liu X. Expanded retroauricular skin and fascial flap in congenital microtia reconstruction. Ann Plast Surg 2010;64:428-34.

11. Chen Z, Zhang W, Huang J, Ren J, Zhu Y. Exceedingly expanded retroauricular flaps for microtia reconstruction. J Plast Reconstr Aesthet Surg 2011;64:1448-53.

12. Firmin F. State-of-the-art autogenous ear reconstruction in cases of microtia. Adv Otorhinolaryngol 2010;68:25-52.

13. Dashan Y, Haiyue J, Qinghua Y, Bo P, Lin L, Tailing W, Yanmei W, Xiao Q, Hongxing Z. Technical innovations in ear reconstruction using a skin expander with autogenous cartilage grafts. J Plast Reconstr Aesthet Surg 2008;61 Suppl 1:S59-69.

14. Jiang H, Pan B, Lin L, Zhao Y, Guo D, Zhuang H. Fabrication of three-dimensional cartilaginous framework in auricular reconstruction. J Plast Reconstr Aesthet Surg 2008;61 Suppl 1:S77-85. 